\title{
Study of cutaneous manifestations of polycystic ovarian syndrome
}

\author{
Sukhleen Kaur ${ }^{1}$, Sunil K. Gupta ${ }^{1}$, Sunil K. Juneja ${ }^{2 *}$, Sukhjot Kaur ${ }^{1}$, Monika Rani ${ }^{1}$
}

${ }^{1}$ Department of Dermatology, Venereology and Leprosy, DMCH, Ludhiana, Punjab, India

${ }^{2}$ Department of Obstetrics and Gynecology, DMCH, Ludhiana, Punjab, India

Received: 29 January 2020

Accepted: 28 February 2020

\section{*Correspondence:}

Dr. Sunil K. Juneja,

E-mail: drsuniljuneja@yahoo.co.in

Copyright: (C) the author(s), publisher and licensee Medip Academy. This is an open-access article distributed under the terms of the Creative Commons Attribution Non-Commercial License, which permits unrestricted non-commercial use, distribution, and reproduction in any medium, provided the original work is properly cited.

\begin{abstract}
Background: Polycystic ovarian syndrome (PCOS) is one of the most frequently encountered endocrine disorders that occurs in as many as 4 to $10 \%$ of women of reproductive age group. It presents with a series of skin changes including acne, hirsutism, seborrhea, androgenetic alopecia (AGA) and acanthosis nigricans. Aim of the study was to determine the prevalence and frequency of different cutaneous manifestations in PCOS patients and to correlate them with the degree of hormonal abnormalities.

Methods: A total 100 patients with features of PCOS who presented to department of dermatology, gynecology (January 2018-December 2019) with cutaneous manifestations were recorded and diagnosis of PCOS was made using Rotterdam's criteria. Pregnant women and diagnosed cases of any other endocrine disorder were excluded. Hirsutism was assessed using Ferriman-Gallwey score and AGA according to Ludwig's classification. Serum hormonal profile including FSH, LH, prolactin, testosterone (free), DHEAS, TSH, FBS, fasting insulin were done. Insulin resistance was determined by calculating HOMA-IR score.

Results: Among cutaneous manifestations of PCOS, hirsutism (85\%) was the most common finding followed by acne (73\%), seborrhea (50\%), AGA (36\%), acanthosis nigricans (29\%) and acrochordons (9\%). The most common hormonal abnormality was insulin resistance in $53 \%$ patients, followed by raised free testosterone in $19 \%$ and serum prolactin in $18 \%$ patients. A statistically significant association was present between AGA and insulin resistance, hirsutism and raised prolactin levels, seborrhea and raised body mass index $(\mathrm{p}<0.05)$.

Conclusions: Dermatological manifestations of PCOS play a significant role in making the diagnosis and constitute a substantial portion of the symptoms experienced by women with this syndrome.
\end{abstract}

Keywords: Acne, Acanthosis nigricans, Androgenetic alopecia, Hirsutism, Polycystic ovarian syndrome, Seborrhea

\section{INTRODUCTION}

Polycystic ovarian syndrome (PCOS) is one of the most frequently encountered endocrine disorders that occurs in as many as 4 to $10 \%$ of women of reproductive age group. ${ }^{1}$ The excessive secretion of androgens in PCOS patients results in a series of skin changes including hirsutism, acne, seborrhea, androgenetic alopecia, acanthosis nigricans and acrochordons. ${ }^{2}$

A revised definition of PCOS was proposed in 2003 at an international joint consensus meeting of the European
Society for Human Reproduction and Embryology and the American Society for Reproductive Medicine. ${ }^{3,4}$

Hirsutism is defined as excessive growth of terminal hair in androgen-dependent areas in women. Acne is found in a large number of PCOS patients as inflammatory lesions on the lower face, neck, chest, and upper aspect of the back. Although sole presence of acne is a potential marker of hyperandrogenism, it is clear that most acne patients do not have androgen excess. ${ }^{5}$ Changes in physical appearance, such as hirsutism and obesity, seem to play the greatest role in the psychosocial 
manifestations. ${ }^{6}$ Early diagnosis and early treatment may prevent metabolic complications and the psychological impact that may negatively impact the patient's quality of life.

Hence, the aim of this study was to determine the pattern and frequency of different cutaneous manifestations in PCOS patients and to correlate them with the hormonal profile.

\section{METHODS}

A cross sectional study with a total 100 patients with features suggestive of PCOS attending department of dermatology, venereology and leprosy and department of obstetrics and gynecology in a tertiary care Hospital of Ludhiana were screened.

\section{Inclusion criteria}

- All female patients with cutaneous manifestations of PCOS

- Menarche to menopause.

\section{Exclusion criteria}

- Pregnant women

- Diagnosed case of any other endocrine disorder (eg. hypothyroidism, hyperprolactinemia, congenital adrenal hyperplasia)

- Patients taking medications like oral contraceptive pills

- Patients unwilling to participate in the study.

A detailed history of patients including age, marital status, family history, menstrual history, treatment history and history of infertility was taken. The body mass index (BMI) was calculated using the formula [weight $(\mathrm{kg}) /$ height $\left.(\mathrm{m})^{2}\right]$. Waist:hip ratio (WHR) was calculated with $\geq 0.85$ considered to be abnormal.

Hirsutism was assessed using Ferriman Gallwey (F-G) score, quantitating the presence of terminal hairs over nine body areas (i.e. upper lip, chin, chest, upper and lower abdomen, upper and lower back, upper arms and thighs). Hirsutism was defined as a Ferriman and Gallwey score $>8$. Androgenetic alopecia was evaluated according to Ludwig's classification.

To diagnose PCOS, patient's pelvic ultrasonography was done mid cycle. Hormonal profile was done on day 2 of menstrual cycle. For amenorrhoeic patients, blood was drawn on any day of the cycle. Fasting venous blood was drawn for FBS, FSH, LH, free testosterone, DHEAS, $\mathrm{TSH}$, fasting insulin, prolactin levels.

Insulin resistance was determined by calculating Homeostatic model assessment of Insulin resistance (HOMA-IR) score.
HOMA-IR $=$ fasting glucose $(\mathrm{mg} / \mathrm{dL}) \times$ fasting insulin $(\mu \mathrm{IU} / \mathrm{mL}) / 405$

Values $>2.5$ were taken as compatible with significant insulin resistance.

Diagnosis of PCOS was made using Rotterdam's criteria.

\section{Statistical analysis}

For comparing categorical data, Chi square $(\chi 2)$ test was performed and exact test was used when the expected frequency is less than 5. A probability value ( $p$ value) less than 0.05 was considered statistically significant. All statistical calculations were done using SPSS 21 (Statistical Package for the Social Science).

\section{RESULTS}

A total 100 female patients diagnosed to have PCOS according to Rotterdam's criteria were included in the study. Results obtained were tabulated and analysed. Mean age of incidence was 23.04 years (Table 1).

Table 1: Age distribution.

\begin{tabular}{|l|l|l|}
\hline Age group & No. of cases & Percentage \\
\hline $17-20$ & 38 & $38 \%$ \\
\hline $21-30$ & 55 & $55 \%$ \\
\hline $31-40$ & 7 & $7 \%$ \\
\hline Total & 100 & $100 \%$ \\
\hline
\end{tabular}

Ultrasonological evidence of PCOS changes was present in $90 \%$ patients (Table 2).

Table 2: Ultrasonological changes.

\begin{tabular}{|ll|l|}
\hline USG & No. of cases & Percentage \\
\hline PCOS & 90 & $90 \%$ \\
\hline NO E/O PCOS & 10 & $10 \%$ \\
\hline Total & 100 & $100 \%$ \\
\hline
\end{tabular}

Table 3: Percentage of cutaneous manifestations.

\begin{tabular}{|lll|}
\hline Cutaneous manifestation & No. of cases & Percentage \\
\hline Hirsutism & 85 & $85 \%$ \\
\hline Acne & 73 & $73 \%$ \\
\hline Seborrhea & 50 & $50 \%$ \\
\hline AGA & 36 & $36 \%$ \\
\hline Acanthosis nigricans & 29 & $29 \%$ \\
\hline Acrochordons & 9 & $9 \%$ \\
\hline
\end{tabular}

A total $82 \%$ patients had h/o irregular menses and $18 \%$ patients had regular menses. The mean BMI in the PCOS patients was $23 \mathrm{~kg} / \mathrm{m}^{2}$ with standard deviation of 2.781 . $48 \%$ patients had normal BMI. 52\% females had increased BMI, including $28 \%$ being overweight and $24 \%$ being obese. 
Table 4: Increased hormone levels in the study group.

\begin{tabular}{|ll|}
\hline Hormone levels & Percentage of patients \\
\hline FSH & $5 \%$ \\
\hline LH & $17 \%$ \\
\hline LH/FSH & $16 \%$ \\
\hline Free testosterone & $19 \%$ \\
\hline DHEAS & $5 \%$ \\
\hline S. Prolactin & $18 \%$ \\
\hline TSH & $7 \%$ \\
\hline HOMA IR & $53 \%$ \\
\hline
\end{tabular}

A total $33 \%$ patients had Waist:Hip ratio more than 0.85 and hence increased risk of metabolic syndrome whereas $67 \%$ patients had Waist:Hip ratio less than 0.85 .

Table 3 shows the percentage of various cutaneous manifestations with hirsutism being the most common manifestation.

The most common hormonal abnormality in the study patients was insulin resistance in $53 \%$ patients, followed by raised free testosterone in $19 \%$ patients and serum prolactin in $18 \%$ patients (Table 4 ).

Table 5 shows correlation of skin changes with hormonal changes. Hormonal levels were raised in almost all the patients with clinical manifestations of PCOS.

Table 6 shows the presence of a statistically significant association between hirsutism and serum prolactin levels. Patients with hirsutism $(n=85)$ were found to have raised serum prolactin levels in $21.2 \%$ patients with a $p$ value of 0.049 . No statistically significant association was seen between hirsutism and other hormone levels (FSH, LH, free testosterone, DHEAS, TSH, FBS, fasting insulin).

Table 7 shows a statistically significant association was also present between seborrhea and BMI. Patients with seborrhea $(n=50)$ had higher BMI indicating obesity in $12 \%$ patients and $32 \%$ patients being overweight with a $p$ value of 0.019 . No statistically significant association was seen between seborrhea and other hormone levels ( $\mathrm{p}$ value $>0.05$ ).

Table 5: Correlation of cutaneous manifestations with increased hormone levels.

\begin{tabular}{|l|lllllllll|}
\hline Cutaneous manifestations & FSH & LH & LH/FSH & Testosterone (free) & DHEAS & Prolactin & TSH & HOMA-IR \\
\hline Hirsutism & 5 & 15 & 15 & 15 & 5 & 18 & 6 & 48 \\
\hline Acne & 2 & 12 & 11 & 16 & 5 & 14 & 6 & 42 \\
\hline Seborrhea & 2 & 10 & 10 & 13 & 3 & 10 & 2 & 31 \\
\hline AGA & 1 & 7 & 9 & 7 & 1 & 9 & 2 & 24 \\
\hline AN & 3 & 2 & 3 & 6 & 2 & 4 & 3 & 17 \\
\hline Acrochordons & 0 & 1 & 1 & 3 & 0 & 3 & 0 & 6 \\
\hline
\end{tabular}

Table 6: Correlation of hirsutism with prolactin level.

\begin{tabular}{|c|c|c|c|c|c|c|c|c|}
\hline \multicolumn{6}{|c|}{ Hirsutism } & \multirow{2}{*}{ Total } & \multirow{2}{*}{ Chi-square value } & \multirow{2}{*}{ p value } \\
\hline & & Yes & & & 15) & & & \\
\hline S. Prolactin & Raised & 18 & $21.2 \%$ & 0 & $0.0 \%$ & 18 & 3.874 & 0.049 \\
\hline
\end{tabular}

Table 7: Correlation of seborrhea with body mass index.

\begin{tabular}{|c|c|c|c|c|c|c|c|c|}
\hline & & \multicolumn{4}{|c|}{ Seborrhea } & \multirow{2}{*}{ Total } & \multirow{2}{*}{ Chi-square value } & \multirow{2}{*}{ p value } \\
\hline & & Ye & $=50)$ & No & & & & \\
\hline \multirow{3}{*}{ BMI group } & Normal & 28 & $56.0 \%$ & 20 & $40.0 \%$ & 48 & \multirow{3}{*}{7.905} & \multirow{3}{*}{0.019} \\
\hline & Obesity & 6 & $12.0 \%$ & 18 & $36.0 \%$ & 24 & & \\
\hline & Overweight & 16 & $32.0 \%$ & 12 & $24.0 \%$ & 28 & & \\
\hline
\end{tabular}

Table 8: Correlation of aga with insulin resistance of women in the study group.

\begin{tabular}{|c|c|c|c|c|c|c|c|}
\hline & & \multicolumn{3}{|l|}{ AGA } & \multirow{2}{*}{ Total } & \multirow{2}{*}{ Chi-square value } & \multirow{2}{*}{ p value } \\
\hline & & Yes $(n=36)$ & No & 64) & & & \\
\hline HOMA-IR & Raised & $66.7 \%$ & 29 & $45.3 \%$ & 53 & 4.218 & 0.04 \\
\hline
\end{tabular}

Table 8 depicts there was a statistically significant association between AGA and insulin resistance. Patients with AGA ( $n=36)$ were found to have raised HOMA - IR scores occurring in $66.7 \%$ patients with a p value of 0.04 . No statistically significant association was seen between 
acne, acanthosis nigricans, acrochordons and hormone levels ( $\mathrm{p}$ value $>0.05$ ).

\section{DISCUSSION}

Patients with PCOS presented to us with hirsutism and acne being their main concern with additional features of androgenetic alopecia, seborrhea, acanthosis nigricans and acrochordons.

The present study comprised of 100 female patients diagnosed to have PCOS according to Rotterdam's criteria and the pattern and frequency of cutaneous manifestations and their correlation with hormonal abnormalities was evaluated and compared.

In this study, mean age of incidence was 23.04 years which was comparable with the studies carried out by Keen et al, Jain et al, while in a study by Mukkamala et al, mean age of incidence was 12.8 years. ${ }^{7-9}$

In present study, $82 \%$ had history of irregular menses and $18 \%$ patients had regular menses which is comparable to other studies. $^{7,9,10}$

In this study, $28 \%$ of the patients were overweight and $24 \%$ were obese while in study by Ramanand et al, obesity was present in $54 \%$ patients and $16 \%$ patients were overweight, in study by Keen et al, obesity was present in $27 \%$ patients and $53 \%$ patients were overweight. ${ }^{7,10}$

Among cutaneous manifestations of PCOS, hirsutism was the most common finding occurring in $85 \%$ patients similar to study by Keen et al and Jain et al. ${ }^{7,8}$ In contrast studies by Gowri et al, Hong et al, Fang et al showed acne to be the most common manifestation. ${ }^{2,11,12}$ Mukkamala et al, found acanthosis nigricans as the most common presentation in cases of PCOS. ${ }^{9}$

The most common hormonal abnormality in this study was insulin resistance in 53\% patients, followed by raised free testosterone in $19 \%$ patients whereas in study by Gowri et al, raised testosterone levels in $55 \%$ patients was the most common manifestation. ${ }^{2}$ In study by Keen et al, raised $\mathrm{LH} / \mathrm{FSH}$ in $38 \%$ patients followed by testosterone levels in $28 \%$ patients were the most common manifestations. $^{7}$

In this study, a statistically significant association was present between hirsutism and serum prolactin levels. Raised prolactin levels were found in $5 \%$ patients in study by Keen et al, $13 \%$ by Spanda et al, and $8 \%$ by Jain et al. ${ }^{7,8,13}$ However no significant association was found between hirsutism and serum prolactin levels in these studies.

No statistically significant association was seen between acne and hormone levels ( $\mathrm{p}$ value $>0.05$ ) in present study while Emiroğlu et al, investigated the presence of insulin resistance in patients with severe acne vulgaris and found a positive correlation between insulin resistance and severe acne vulgaris. ${ }^{14}$

A statistically significant association was also present between seborrhea and BMI. Patients with seborrhea (n50 ) had higher BMI indicating obesity in $12 \%$ patients and $32 \%$ patients being overweight.

In a study by Al-Saeed et al, there was increased prevalence of seborrhoea in obese patients which was seen in $23.8 \%$ patients. $^{15}$ No significant association between obesity and seborrhea has been recorded in PCOS patients in other studies.

In present study, there was a statistically significant association between AGA and insulin resistance similar to study by Bakry et al, Matilainen et al, also reported a significantly higher value of fasting insulin in AGA cases than in the control group. ${ }^{16,17}$

\section{CONCLUSION}

Polycystic ovarian syndrome (PCOS) is one of the most frequently encountered endocrine disorders in women of reproductive age group.

The evaluation should include detailed menstrual history, information about the onset and duration of symptoms suggestive of hyperandrogenism and family history of PCOS and metabolic diseases.

Dermatological manifestations may provide early clinical clues to the recognition of PCOS, and treatment of these cutaneous conditions may improve the patient's quality of life and psychological well-being. If history and physical examination do not delineate the underlying cause, laboratory testing can be helpful. Other etiologies of hyperandrogenism (congenital adrenal hyperplasias, androgen -secreting tumours, Cushing's syndrome) should be ruled out.

So, authors recommend performing endocrinological work up, investigation of coexisting hyperandrogenic states, and evaluation of polycystic ovary syndrome in all patients with hirsutism. PCOS has many potential metabolic and cardiovascular risks if not managed appropriately, thus proper diagnosis and management is essential.

\section{Funding: No funding sources}

Conflict of interest: None declared

Ethical approval: The study was approved by the Institutional Ethics Committee

\section{REFERENCES}

1. Goodarzi MO, Azziz R. Diagnosis, epidemiology, and genetics of the polycystic ovary syndrome. Best Pract Res Clin Endocrinol Metab. 2006;20:193-205. 
2. Gowri BV, Chandravathi PL, Sidhu PS, Naidu KS. Correlation of skin changes with hormonal changes in polycystic ovarian syndrome: a cross-sectional study clinical study. Indian J Dermatol. 2015;60:419.

3. Revised 2003 consensus on diagnostic criteria and long-term health risks related to polycystic ovary syndrome (PCOS). Hum Reprod. 2004;19(1):41-7.

4. Revised 2003 consensus on diagnostic criteria and long-term health risks related to polycystic ovary syndrome. Fertil Steril. 2004;81(1):19-25.

5. Archer JS, Chang RJ. Hirsutism and acne in polycystic ovary syndrome. Best Pract Res Clin Obstet Gynaecol. 2004;18:737-54.

6. Clark AM, Ledger W, Galletly C, Tomlinson L, Blaney $\mathrm{F}$, Wang $\mathrm{X}$, et al. Weight loss results in significant improvement in pregnancy and ovulation rates in anovulatory obese women. Hum Reprod. 1995; 10:2705-12.

7. Keen MA, Shah IH, Sheikh G. Cutaneous manifestations of polycystic ovary syndrome: a cross-sectional clinical study. Indian J Dermatol. 2017;8:104-10.

8. Jain P, Jain SK, Singh A, Goel S. Pattern of dermatologic manifestations in polycystic ovarian disease cases from a tertiary care hospital. Int $\mathrm{J} \mathrm{Adv}$ Med. 2018;5:197-201.

9. Mukkamala S, Aruna C, Ramamurthy DV, Sridevi K, Senthil AL, Kameti S. Cutaneous manifestations in polycystic ovarian syndrome: a clinicoepidemiological study. J Pak Assoc Dermatol. 2019;28:410-4.

10. Ramanand SJ, Ghongane BB, Ramanand JB, Patwardhan MH, Ghanghas RR, Jain SS. Clinical characteristics of polycystic ovary syndrome in Indian women. Indian $\mathrm{J}$ Endocrinol Metabol. 2013; $17: 138$.

11. Hong JS, Kwon HH, Park SY, Jung JY, Yoon JY, Min S, et al. Cutaneous manifestations of the subtypes of polycystic ovary syndrome in Korean patients. J Eur Acad Dermatol Venereol. 2015;29:427.

12. Feng JG, Guo Y, Ma LA, Xing J, Sun RF, Zhu W. Prevalence of dermatologic manifestations and metabolic biomarkers in women with polycystic ovary syndrome in north China. J Cosmet Dermatol. 2018;17:511-7.

13. Spandana JC, Shetty PK. A study on the clinical, biochemical and hormonal profile of polycystic ovary syndrome patients attending tertiary care hospital. Int J Reprod Contracept Obstet Gynecol. 2017;6:1986-92.

14. Emiroğlu N, Cengiz FP, Kemeriz F. Insulin resistance in severe acne vulgaris. Postep Derm Alergol. 2015;XXXII:281-5.

15. Al-Saeed WY, Al-Dawood KM, Bukhari IA, Bahnassy A. Dermatoses in obese female schoolchildren in the Al-Khobar area, Eastern Saudi Arabia. J Family Community Med. 2006;13:65-9.

16. Bakry OA, Shoeib MA, El Shafiee MK, Hassan A. Androgenetic alopecia, metabolic syndrome, and insulin resistance: Is there any association? A casecontrol study. Indian Dermatol Online J. 2014;5:27681.

17. Matilainen V, Koskela P, Keinanen-Kiukaanniemi S. Early and rogenetic alopecia as a marker of insulin resistance. Lancet. 2000;356:1165-6.

Cite this article as: Kaur S, Gupta SK, Juneja SK, Kaur S, Rani M. Study of cutaneous manifestations of polycystic ovarian syndrome. Int J Reprod Contracept Obstet Gynecol 2020;9:1627-31. 